OPEN ACCESS

Edited by:

Sikha Saha,

University of Leeds, United Kingdom

Reviewed by:

Margarida Castro-Caldas, Universidade Nova de Lisboa,

Portugal

Federico Herrera, Instituto de Tecnologia Química e Biológica (ITQB-NOVA), Portugal

*Correspondence: Yewei Sun yxy0723@163.com

Zaijun Zhang zaijunzhang@163.com

Specialty section: This article was submitted to

Neurodegeneration,

a section of the journal

Frontiers in Neuroscience

Received: 27 December 2017

Accepted: 27 July 2018

Published: 15 August 2018

Citation:

Chen H, Cao J, Zhu Z, Zhang G,

Shan L, Yu P, Wang Y, Sun Y and

Zhang $Z$ (2018) A Novel

Tetramethylpyrazine Derivative

Protects Against Glutamate-Induced Cytotoxicity Through PGC1 a/Nrf2 and PI3K/Akt Signaling Pathways.

Front. Neurosci. 12:567.

doi: 10.3389/fnins.2018.00567

\section{A Novel Tetramethylpyrazine Derivative Protects Against Glutamate-Induced Cytotoxicity Through PGC1 $\alpha /$ Nrf2 and PI3K/Akt Signaling Pathways}

\author{
Haiyun Chen ${ }^{1,2}$, Jie Cao ${ }^{2}$, Zeyu Zhu², Gaoxiao Zhang ${ }^{2}$, Luchen Shan², Pei Yu², \\ Yuqiang Wang ${ }^{2}$, Yewei Sun ${ }^{2 *}$ and Zaijun Zhang ${ }^{2 *}$
}

\begin{abstract}
${ }^{1}$ Institute of Biomedical and Pharmaceutical Sciences, Guangdong University of Technology, Guangzhou, China, ${ }^{2}$ Institute of New Drug Research and Guangzhou Key Laboratory of Innovative Chemical Drug Research in Cardio-Cerebrovascular Diseases, Jinan University College of Pharmacy, Guangzhou, China
\end{abstract}

Glutamate-induced excitotoxicity is one of the main causes of neuronal cell death in stroke. Compound 22a has been previously reported as a promising neuroprotective compound derived from tetramethylpyrazine, which is a widely used active ingredient of traditional Chinese medicine Chuanxiong (Ligusticum wallichii Franchat). Compound 22a can protect neurons from oxidative stress-induced PC12 cell death and alleviates the infarct areas and brain edema in a rat permanent middle cerebral artery occlusion model. In the current work, we further investigated the neuroprotective effects and underlying mechanisms of compound $\mathbf{2 2 a}$ against glutamate-induced excitotoxicity in primary culture of rat cerebellar granule neurons (CGNs). We found that pretreatment with compound 22a prevented glutamate-induced neuronal damage by maintaining mitochondrial membrane potential and attenuating cellular apoptosis. Compound 22a could also enhance peroxisome proliferator-activated receptor gamma coactivator 1alpha (PGC1 $\alpha$ ) transcriptional activity and induce nuclear accumulation of Nrf2 in PC12 cells. Accordingly, pretreatment with compound 22a reversed the glutamate-induced down-regulation of expression of the proteins PGC1 $1 \alpha$, transcriptional factor NF-E2related factor 2 (Nrf2), and hemooxygenase 1 (HO-1). In addition, compound 22a increased the phosphorylation of phosphoinositide 3-kinase (p-PI3K), phosphorylated protein kinase B (p-Akt), and glycogen synthase kinase $3 \beta$ (p-GSK3 $\beta$ ). Meanwhile, the small interfering RNA-mediated silencing of PGC1 $\alpha$ expression and selective inhibitors targeting PI3K/Akt (LY294002 and Akt-iv) could significantly attenuate the neuroprotective effect of compound 22a. Taken together, compound 22a protected against glutamate-induced CGN injury possibly in part through regulation of PGC1 $\alpha / \mathrm{Nrf2}$ and PI3K/Akt pathways.

Keywords: ischemic stroke, tetramethylpyrazine derivative 22a, excitotoxicity, neuroprotection, peroxisome proliferator-activated receptor gamma coactivator 1-alpha

Abbreviations: Akt, protein kinase B; CGN, cerebellar granule neurons; Cyt C, cytochrome c; GSK3 $\beta$, glycogen synthase

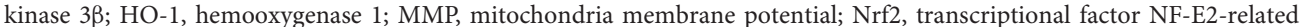
factor 2; PGC1 $\alpha$, peroxisome proliferator-activated receptor gamma coactivator 1-alpha; PI3K, phosphatidylinositol 3-kinase; ROS, reactive oxygen species; TMP, tetramethylpyrazine. 


\section{INTRODUCTION}

Ischemic stroke is one of the major causes of human death and disability worldwide (Donnan et al., 2008). Glutamate-induced excitotoxicity has been demonstrated to be involved in neuronal cell death in stroke (Lai et al., 2014). Physiologically, glutamate acts as one of the main excitatory neurotransmitters in the central nervous system (CNS), contributing to normal neural transmission, development, differentiation, and plasticity. Under pathological conditions, however, overproduction of extracellular glutamate leads to uncontrolled, continuous depolarization of neurons in a toxic process called excitotoxicity. Glutamateinduced excitotoxicity is associated with the over-stimulation of glutamate receptors, inducing the impairment of intracellular $\mathrm{Ca}^{2+}$ homeostasis and subsequently leading to overproduction of free radicals, overactivation of proteases and kinases, etc. (Wang and Qin, 2010; Lai et al., 2014). Particularly, the overloading intracellular $\mathrm{Ca}^{2+}$ and overproduction of free radicals have been shown to induce mitochondrial dysfunction by down-regulating PGC1 $\alpha$, which plays a protective role against neurodegenerative conditions (Sano and Fukuda, 2008; Wareski et al., 2009). Although great progress has been made, the exact mechanism underlying glutamate-induced cytotoxicity is still not clear. Nonetheless, it has been reported that dysregulation of PI3K/Akt and Nrf2 signaling pathways contributes to glutamate-induced excitotoxicity (Jing et al., 2012; Pang et al., 2016). Stimulation of the PI3K/Akt pathway is neuroprotective against hypoxic and excitotoxic neuronal death in vitro and ischemic neuronal death in vivo, and there is increasingly evidence to indicate cross talk between the Nrf2 and PI3K/Akt pathways in response to glutamate caused cell injury (Jo et al., 2012; Lee et al., 2015).

Since the exact causes of ischemic stroke have yet to be elucidated, currently there are no pharmacological treatments to ameliorate glutamate excitotoxicity and provide neuroprotection for brain ischemic stroke (Lau and Tymianski, 2010). Thrombolysis via the intravenous (i.v.) administration of recombinant tissue plasminogen activator remains the only treatment currently available for acute ischemic stroke. In addition, it is of great concern that clinical trials investigating neuroprotective agents for the development of new stroke therapies have generally been unsuccessful (Grupke et al., 2014). As a result, numerous researchers have been exploring potentially active plant-derived agents, hoping to meet this unmet need and discover a disease-modifying drug (Zhang et al., 2014). Traditional Chinese medicine focuses on the overall regulation of the pathophysiological condition of the entire body, a trait that makes these compounds particularly promising in the treatment of complex diseases (Ghosh et al., 2014). TMP is one of the mainly biologically active constituents derived from the traditional Chinese medicine Chuanxiong (Ligusticum wallichii Franchat) and has been widely used to treat cardioand cerebro-vascular diseases in clinic (Liu et al., 2003; Xue et al., 2011). In our previous study, a TMP-derived compound 22a was designed to combine caffeic acid (Touaibia et al., 2011) (another natural compound with versatile pharmacological activities) and a nitrone group (Floyd et al., 2013) (a strong free radical-trapping agent) with TMP. We found that compound 22a exhibited strong ROS scavenging activity and exerted protective effects in models of ischemic stroke in vivo (Chen et al., 2017). In the present study, the neuroprotective effects of compound 22a against glutamate-induced excitotoxicity on primary culture of rat CGNs, and the underlying mechanisms of action, were further investigated.

\section{MATERIALS AND METHODS}

\section{Chemicals and Reagents}

All media and supplements used for cell cultures were purchased from Gibco (Carlsbad, CA, United States), unless otherwise noted. ATP assay kit was obtained from Beyotime (Beyotime, China). LY294002 and Akt-iv was obtained from Sigma-Aldrich (St. Louis, MO, United States). PGC1 $\alpha$ siRNA, scrambled siRNA and transfection reagent were purchased from Santa Cruz Biotechnology (Santa Cruz, CA, United States). RIPA lysis buffer, phenylmethanesulfonyl fluoride (PMSF), and halt phosphatase inhibitor cocktail were purchased from Pierce Biotechnology (Rockford, IL, United States). Antibodies against phospho-Ser473 Akt, phospho-Ser9 GSK3 $\beta, \mathrm{Bcl}-2$ and Bax were obtained from Cell Signaling Technology (Beverly, MA, United States). Antibodies against $\beta$-actin, $\mathrm{PGCl} \alpha, \mathrm{Nrf} 2$ and $\mathrm{HO}-$ 1 were obtained from Santa Cruz Biotechnology (Santa Cruz, CA, United States). All other reagents were from Sigma-Aldrich (St. Louis, MO, United States) except where stated otherwise.

\section{Primary Cell Cultures}

Eight-day-old Sprague-Dawley rats were obtained from the Experimental Animal Center of Sun Yat-sen University. The detailed methodology used to separate the CGNs is described in our previous publication (Chen H.Y. et al., 2015). Briefly, neurons were seeded at a density of 1.0-1.5 $\times 10^{5}$ cells/well in basal modified Eagle's medium supplemented with $10 \%$ fetal bovine serum, $25 \mathrm{mM} \mathrm{KCl}, 2 \mathrm{mM}$ glutaMax and penicillin (100 $\mathrm{U} / \mathrm{mL}) /$ streptomycin $(100 \mu \mathrm{g} / \mathrm{mL})$. The cultures were grown at $37^{\circ} \mathrm{C}$ for $24 \mathrm{~h}$. The growth of non-neuronal cells was limited by adding cytosine arabinoside $(10 \mu \mathrm{M})$. Using this protocol, 95$99 \%$ of the cultured cells were granule neurons. All experiments were performed in CGNs at 8 days in vitro (DIV).

All experiments were conducted in accordance with the guidelines of the Experimental Animal Care and Use Committee of Jinan University. The experimental protocols were approved by the Ethics Committee for Animal Experiments of Jinan University.

\section{MTT Reduction Assay}

The tetrazolium salt 3-(4,5-dimethylthiazol-2-yl)-2,5diphenyltetrazolium bromide dye (MTT) assay was used to assess neurotoxicity. The assay was performed according to the procedure described in our previous work (Chen H.Y. et al., 2015). Briefly, neurons were cultured in 96-well plates for 8 DIV. Treatments of compound 22a $(0.1,1,10,100 \mu \mathrm{M})$, TMP $(100 \mu \mathrm{M})$ and memantine $(5 \mu \mathrm{M})$ were used as controls for $2 \mathrm{~h}$ before $200 \mu \mathrm{M}$ glutamate was added to the media. To determine the possible pathways involved in the effect of 
compound 22a, CGNs were pretreated with $1 \mu \mathrm{M}$ LY294002 (a PI3K inhibitor) or $1 \mu \mathrm{M}$ Akt-iv (an Akt inhibitor) for $30 \mathrm{~min}$ before administration of compound 22a $(10 \mu \mathrm{M})$, and were then challenged by glutamate. After $24 \mathrm{~h}$, the media of each well was supplemented with $10 \mu \mathrm{L}$ of $5 \mathrm{mg} / \mathrm{mL}$ MTT, and the plate was put in an incubator at $37^{\circ} \mathrm{C}$ for $4 \mathrm{~h}$ under humidified conditions. A microplate reader was used to measure the absorbance of the samples at $570 \mathrm{~nm}$ wavelength.

\section{Lactate Dehydrogenase (LDH) Release}

The activity of $\mathrm{LDH}$ released into the incubation medium was used as an indicator to determine cellular injury. CGNs were pretreated with compound 22a $(0.1,1,10,100 \mu \mathrm{M})$ or TMP $(100 \mu \mathrm{M})$ and memantine $(5 \mu \mathrm{M})$ for $2 \mathrm{~h}$, then $200 \mu \mathrm{M}$ glutamate was added to incubated for $24 \mathrm{~h}$. The cytotoxicity detection kit (Roche Applied Science, Germany) was used to determine LDH activity. LDH release was calculated according to the manufacturer's instructions. A microplate reader was used to measure the absorbance at $490 \mathrm{~nm}$ wavelength. Cytotoxicity (\%) was calculated with the following equation:

Cytotoxicity $(\%)=($ experimental LDH release spontaneous LDH release)/ (Maximum LDH release-

$$
\text { spontaneous LDH release)* } 100
$$

Experimental $\mathrm{LDH}$ release represents the activity of released $\mathrm{LDH}$ in cells pretreated with test compounds and glutamate, Spontaneous LDH release represents the activity of released-LDH in untreated normal cells, and Maximum LDH release represents maximum LDH activity determined by lysing the cells $(100 \%$ dead cells) with Triton X-100 (final concentration $2 \%$ for $4 \mathrm{~h}$ at $37^{\circ} \mathrm{C}$ ). All assays were performed in six-replicates and repeated three times.

\section{Hoechst Staining}

Chromatin condensation was detected by nucleus staining with Hoechst 33342 (Beyotime, China) as previously described (Chen H.Y. et al., 2015). CGNs of $4-5 \times 10^{5}$ cells/well were cultured in a 24-well plate for 8 DIV. Pretreatment of compound 22a $(100 \mu \mathrm{M})$ and memantine $(5 \mu \mathrm{M})$ as the positive control at the indicated concentrations for $2 \mathrm{~h}$ before $200 \mu \mathrm{M}$ glutamate was added into the media. After $24 \mathrm{~h}$, cells were washed with ice-cold phosphatebuffered saline (PBS) and fixed with $4 \%$ formaldehyde in PBS. Hoechst $33342(5 \mathrm{mg} / \mathrm{mL})$ was added to the media for $5 \mathrm{~min}$ at $4^{\circ} \mathrm{C}$. Samples were observed and photos were taken under a fluorescence microscope. The fluorescence images were phototaken from three different fields of each experiment with a total of three independent experiments. The number of apoptotic nuclei was counted and expressed as a percentage of total 100 nuclei counted/field.

\section{Measurement of Intracellular Reactive Oxygen Species (ROS)}

Cerebellar granule neurons were cultured in 96-well plates at a density of 1.0-1.5 $\times 10^{5}$ cells/well for $8 \mathrm{DIV}$. After pretreatment with compound $22 \mathrm{a}(0.1,1,10,100 \mu \mathrm{M})$ or memantine $(5 \mu \mathrm{M})$ for $2 \mathrm{~h}$, they were then exposed to $200 \mu \mathrm{M}$ glutamate for $24 \mathrm{~h}$. The total intracellular ROS were detected using $\mathrm{H}_{2}$ DCF-DA $(10 \mu \mathrm{M})$, and a microplate reader was used to measure the fluorescence intensity. The fluorescence intensity/per well was normalized against the MTT absorption value of the same well. The fluorescence values of the treated group were calculated as a percentage of the fluorescence of the control cells.

\section{Measurement of Mitochondrial Membrane Potential}

Cerebellar granule neurons were placed in a 96-well plate at a density of 1.0-1.5 $\times 10^{5}$ cells/well. After $8 \mathrm{DIV}$, compound 22a or memantine was added to pretreat cells at the indicated concentrations for $2 \mathrm{~h}$. After exposure to $200 \mu \mathrm{M}$ glutamate for $24 \mathrm{~h}$, the cells were washed three times and then stained with 2 MM JC-1 (Beyotime, China), a molecular probe to measure mitochondrial membrane potential (MMP), for $10 \mathrm{~min}$. The microplate reader was used to measure the fluorescence intensity using $490 \mathrm{~nm} / 530 \mathrm{~nm}$ excitation and $525 \mathrm{~nm} / 590 \mathrm{~nm}$ dual emissions. The mitochondrial accumulation of JC-1 is dependent upon MMP, which is calculated as a decrease in the ratio of $590 \mathrm{~nm}$ to $525 \mathrm{~nm}$ emissions.

\section{Measurement of Intracellular ATP Levels}

Cerebellar granule neurons were placed in a 96-well plate at a density of 1.0-1.5 × $10^{5}$ cells/well. After 8 DIV, 22a or memantine was added to pretreat at the indicated concentrations for $2 \mathrm{~h}$. After the following incubation with $200 \mu \mathrm{M}$ glutamate for $24 \mathrm{~h}$, a ATP Assay Kit was used to detect the intracellular ATP levels by using the (Beyotime, China) according to the manufacturer's protocol. The intracellular ATP levels of the treated group were normalized to the control cells'.

\section{Western Blot Assay}

Cerebellar granule neurons were cultured in a 6-well plate at a density of $2 \times 10^{6}$ per well in $1.5 \mathrm{~mL}$. At $8 \mathrm{DIV}$, cells were pretreated with 22a for $2 \mathrm{~h}$ before incubation with $200 \mu \mathrm{M}$ glutamate for another $12 \mathrm{~h}$. Cells were harvested in a cell lysis buffer supplemented with 1\% PMSF (phenylmethanesulfonyl fluoride) as well as $1 \%$ protease inhibitor cocktail (Roche Applied Science). The cellular protein concentrations were determined by the BCA assay (Pierce, Rockford, IL, United States) after incubation for $15 \mathrm{~min}$ on ice and centrifugation $(14,000 \mathrm{~g})$ for $10 \mathrm{~min}$ at $4^{\circ} \mathrm{C}$. SDS sample buffer was added to dilute the cell lysates, and the mixture was heated for $5 \mathrm{~min}$ at $100^{\circ} \mathrm{C}$. The protein $(30 \mu \mathrm{g})$ was separated on a $10 \%$ SDS-polyacrylamide gel. After transferring protein to polyvinyldifluoride membranes, $5 \%$ BSA was used as a blocking buffer to block the membranes. After incubation with the primary antibodies at $4^{\circ} \mathrm{C}$ overnight, signals were attained by binding a secondary antibody conjugated to horseradish peroxidase. Blots were detected using the chemiluminescence enhancer detection ECL plus kit (Fude Biological Technology Co., Ltd., China) and images captured using a detecting system (Carestream Health, United States). Quantitation of the digitalized images was analyzed based on their mean pixel density by using Carestream software system, 
and described as an expression ratio of target protein to $\beta$-actin (a loading control protein).

\section{Silencing of PGC1 $\alpha$ by Small Interfering RNA (siRNA)}

Cerebellar granule neurons at 8 DIV were transfected with siRNA using transfection reagent Opti-MEM I (Invitrogen, Carlsbad, CA, United States) and Lipofectamine2000 (Invitrogen) according to the manufacturer's instructions and the previous studies' (Kim et al., 2014). Experiments were carried out $24 \mathrm{~h}$ after the cells were transfected with PGC1 $\alpha$ siRNA or scrambled siRNA.

\section{PGC1 $\alpha$ Transcriptional Activity}

PC12 cells were plated in 24-well plates and transfected with a PGC1 $\alpha$ luciferase reporter plasmid along with Renilla luciferase control vector by using the Amaxa Nucleofector II, which can be used for transfection of any DNA vectors (expression plasmids, shRNA vectors) into difficult-to-transfect cell lines and primary cells. Then $12 \mathrm{~h}$ after transfection, the cells were treated with compound 22a at the indicated concentrations for $24 \mathrm{~h}$. Cells were harvested and analyzed using a Dual-Glo luciferase assay kit (Promega, Madison, WI, United States) following the manufacturer's instructions. Firefly luciferase activity was normalized to Renilla luciferase activity.

\section{Immunofluorescence}

Cerebellar granule neurons of $4-5 \times 10^{5}$ cells/well were cultured in a 24-well plate for $8 \mathrm{DIV}$, and were treated with compound 22a $(100 \mu \mathrm{M})$. After $12 \mathrm{~h}$, cells were washed with ice-cold phosphatebuffered saline (PBS) and fixed with 4\% formaldehyde in PBS for $30 \mathrm{~min}$ at room temperature. The cells were washed with ice-cold PBS containing 5\% BSA acting as a blocking buffer for $1 \mathrm{~h}$ at room temperature before being incubated with anti-Nrf2 antibody (1:500) overnight at $4^{\circ} \mathrm{C}$. The cells were then washed with ice-cold PBS and incubated with FITC antibody (1:200) (Sigma-Aldrich, St. Louis, MO, United States) containing $200 \mathrm{nM}$ DAPI for $2 \mathrm{~h}$ at room temperature. Samples were observed and photos were taken under a fluorescence microscope.

\section{Statistical Analysis}

All experiments were carried out at least three times with different neuronal preparations, data from which was presented as means \pm SEM. Analysis of variance (ANOVA) and Bonferroni's
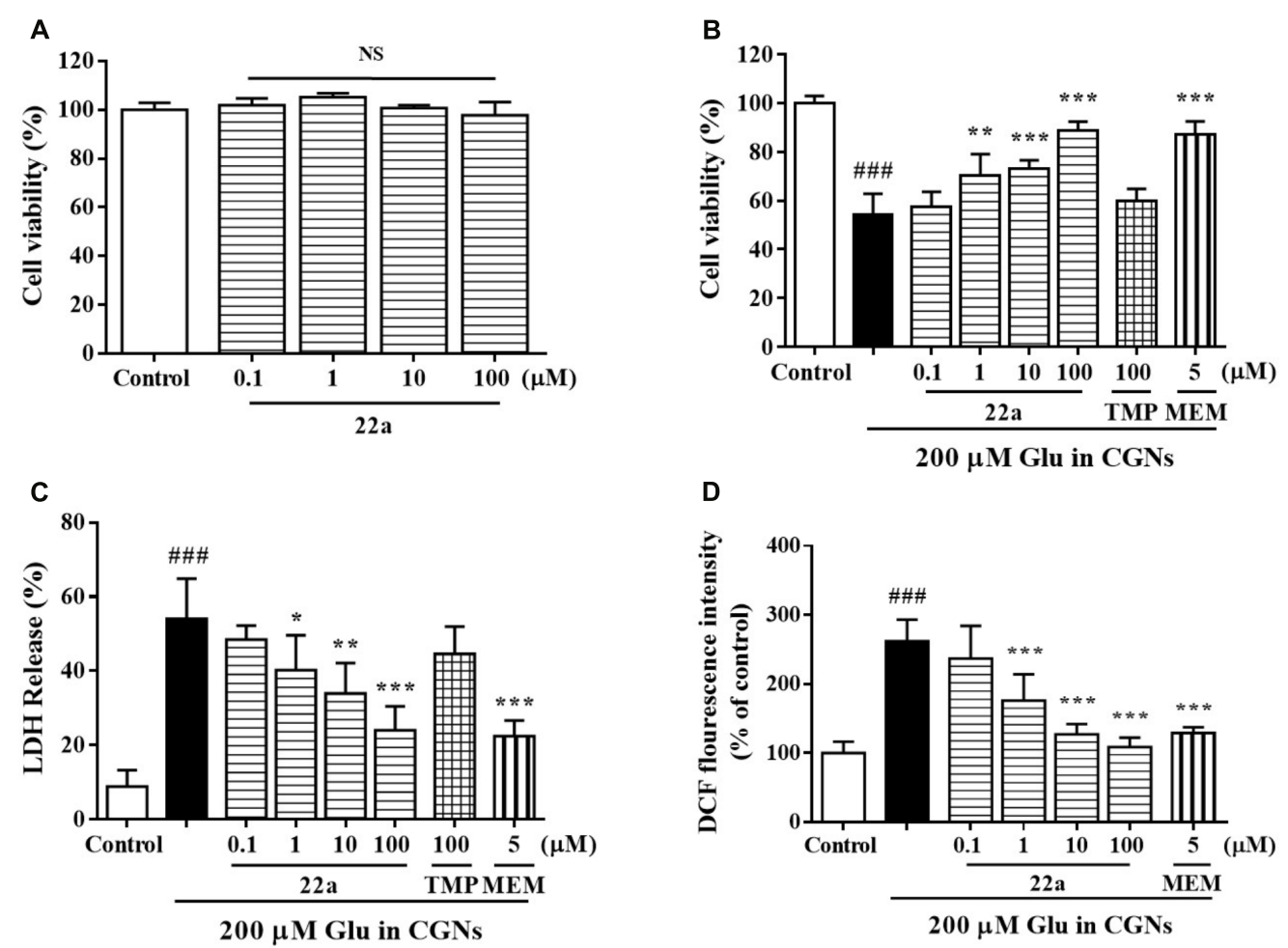

FIGURE 1 | Compound 22a prevents glutamate-induced neurotoxicity. (A) Toxicity of compound $\mathbf{2 2 a}$ on CGNs. CGNs were pre-incubated with compound $\mathbf{2 2} \mathbf{a}$ for 24 h. Cell viability was measured using the MTT assay. (B) Compound 22a prevents glutamate-induced neuronal death in CGNs. CGNs were pre-incubated with various agents for $2 \mathrm{~h}$, then exposed to $200 \mu \mathrm{M}$ glutamate. Cell viability was measured at $24 \mathrm{~h}$ post glutamate challenge using the MTT assay. (C) Compound $\mathbf{2 2 a}$ prevents glutamate-induced increase of LDH in CGNs (\% of control). CGNs were pre-incubated with various agents for $2 \mathrm{~h}$, then exposed to $200 \mathrm{\mu M}$ glutamate. $\mathrm{LDH}$ release was measured at $24 \mathrm{~h}$ after glutamate challenge. (D) Compound 22a attenuates glutamate-induced increase of ROS in CGNs. CGNs were pre-incubated with various compounds for $2 \mathrm{~h}$, then exposed to $200 \mu \mathrm{M}$ glutamate for another $24 \mathrm{~h}$. Intracellular hydrogen peroxide was measured by DCF-DA. Data were expressed as the mean \pm SEM of three separate experiments; ${ }^{\# \# \#} p<0.001$ versus control group; ${ }^{*} p<0.05,{ }^{* *} p<0.01$, and ${ }^{* * *} p<0.001$ versus glutamate group. Ctrl means control. Glu, glutamate. MEM, memantine. 
post-test were used for statistical comparisons, with $P<0.05$ being considered as statistical significance.

\section{RESULTS}

\section{Compound 22a Effectively Prevents Glutamate-Induced Neurotoxicity}

At 8 DIV, CGNs were exposed to increasing concentrations of glutamate, which induced a concentration-dependent neuronal death from 50 to $1000 \mu \mathrm{M}$ (data not shown). Since low doses of glutamate induced apoptosis instead of necrosis in primary CGNs (Du et al., 1997), a $200 \mu \mathrm{M}$ glutamate concentration that produced approximately 54\% neuronal loss was used for the subsequent experiments. Treatment with compound 22a up to $100 \mu \mathrm{M}$ for $24 \mathrm{~h}$ didn't cause any cytotoxicity to CGNs (Figure 1A). Pretreatment with serial concentrations of compound 22a (1-100 $\mu \mathrm{M})$ could prevent glutamate-induced toxicity and the maximum cell viability reached $88.7 \%$ at $100 \mu \mathrm{M}$, similar to that of positive control memantine $(87.1 \%)$ and higher than that of TMP (60.0\%) (Figure 1B). The neuroprotection of compound 22a against glutamate triggered cytotoxicity was more potent than that of TMP.

To further confirm the protection of compound 22a against glutamate-induced neurotoxicity, we measured the $\mathrm{LDH}$ release and intracellular ROS production in CGNs. Compound 22a and memantine significantly prevented glutamate-induced LDH release. TMP $(100 \mu \mathrm{M})$ had a marginal effect in this model (Figure 1C). Furthermore, pretreatment with compound 22a from 1 to $100 \mu \mathrm{M}$ and memantine significantly decreased the intracellular ROS overproduction caused by glutamate in CGNs (Figure 1D).

\section{Compound 22a Inhibits Glutamate-Induced Cellular Apoptosis in CGNs}

It has been reported that a $200 \mu \mathrm{M}$ glutamate concentration induced neuronal damage via apoptosis (Du et al., 1997). Compound 22a and memantine significantly reversed the cell counts of nuclear condensation induced by glutamate (Figures 2A,B). In Figure 2C, two apoptosis related proteins, Bcl-2 and Bax, were determined by Western blot analysis. It was found that pretreatment with compound 22a reversed the Bcl-2 down-regulation and Bax up-regulation induced by glutamate. The $\mathrm{Bcl}-2 / \mathrm{Bax}$ ratio was increased by pretreatment with compound 22a in a concentration-dependent manner (Figure 2D).

\section{Compound 22a Improves the Mitochondrial Dysfunction Induced by Glutamate}

To examine whether the neuroprotective effects of compound 22a was due to preservation of mitochondrial function, we

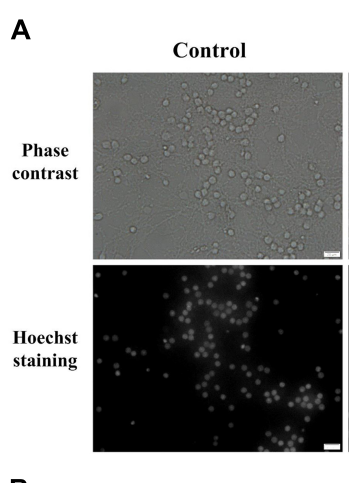

B

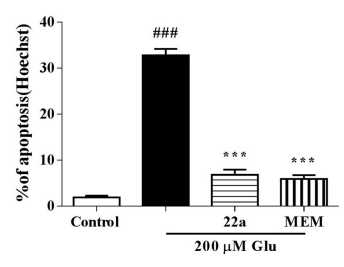

Glu

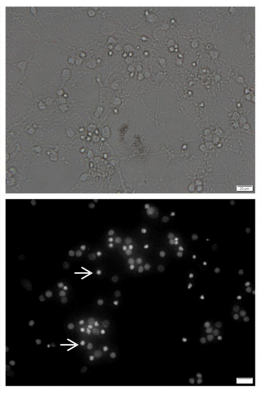

C

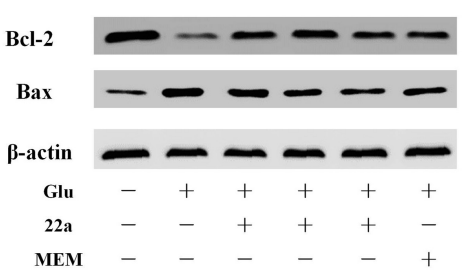

Glu + MEM $(5 \mu M)$
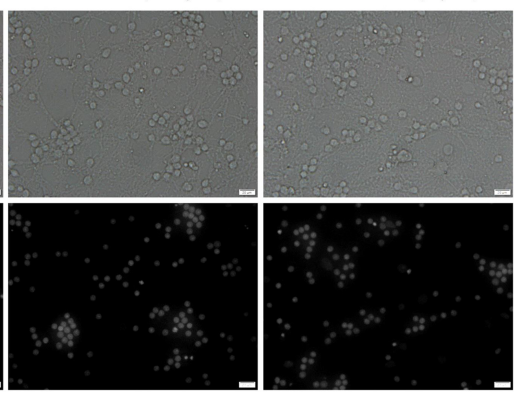

D

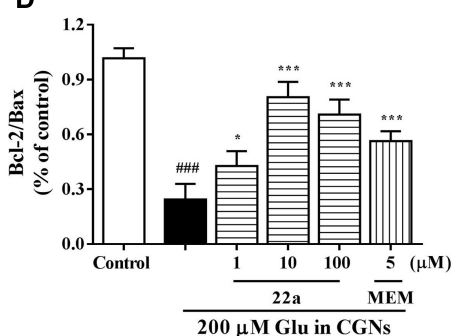

FIGURE 2 | 22a inhibits glutamate-induced cell apoptosis in CGNs. (A) Compound 22a prevents glutamate-induced increase of pyknotic nuclei in CGNs. CGNs were pre-incubated with various agents for $2 \mathrm{~h}$, then exposed to $200 \mu \mathrm{M}$ glutamate. CGNs were stained with Hoechst $24 \mathrm{~h}$ after the glutamate challenge. Photographs were from a representative experiment; experiments were repeated three times (original magnification $400 \times$ ). (B) Statistical analysis of the number of pyknotic nuclei. The number of pyknotic nuclei with condensed chromatin was counted from representative Hoechst staining photomicrographs and is represented as a percentage of the total number of nuclei counted. (C) Western blot of apoptosis related proteins Bcl-2 and Bax. (D) Densitometry analysis of protein expression ratios of Bcl-2 and Bax. Data were expressed as the mean \pm SEM of three separate experiments; ${ }^{\# \# \# ~} p<0.001$ versus control group; ${ }^{*} p<0.05$ and ${ }^{* * *} p<0.001$ versus glutamate group. 
A
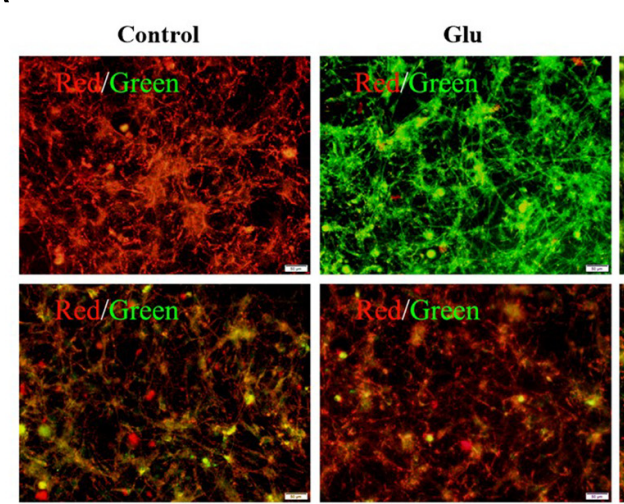

Glu + $10 \mu \mathrm{M} \mathrm{22a}$

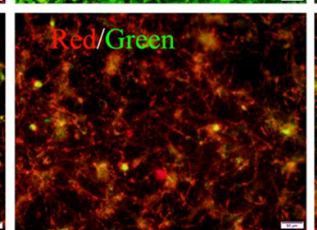

Glu $+100 \mu \mathrm{M} 22 \mathrm{a}$

B

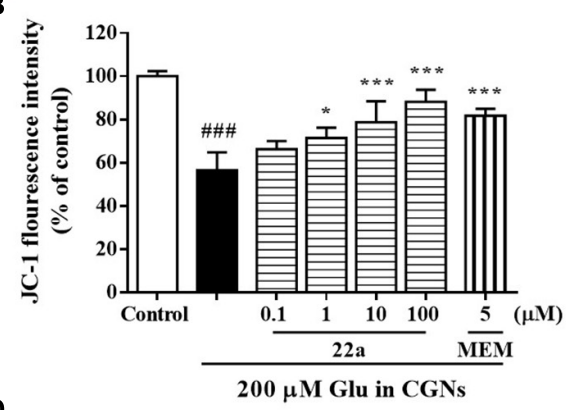

D

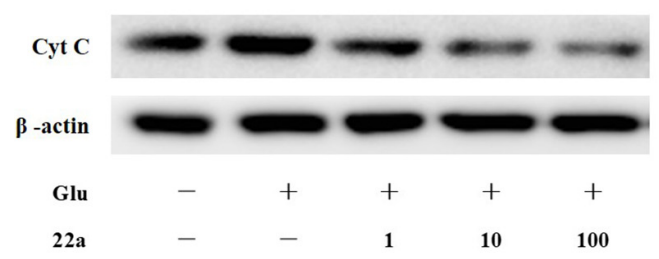

Glu $+0.1 \mu \mathrm{M} \mathrm{22a}$
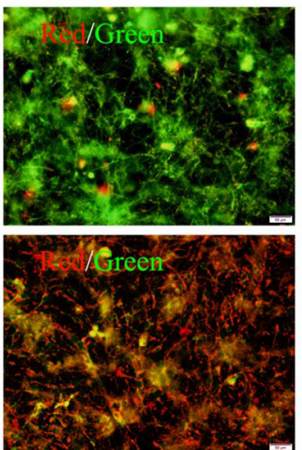

Glu + 5 $\mu$ M MEM

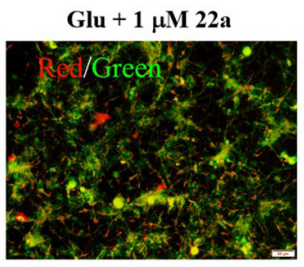

C

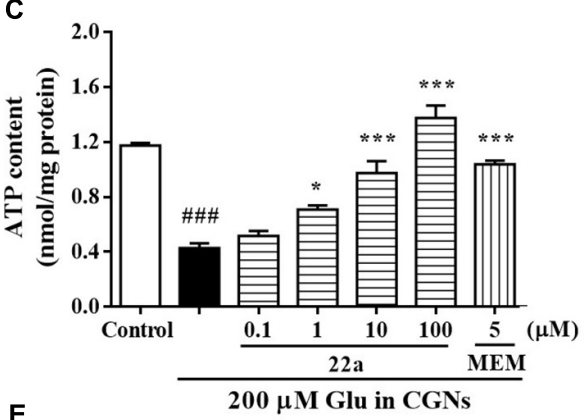

E

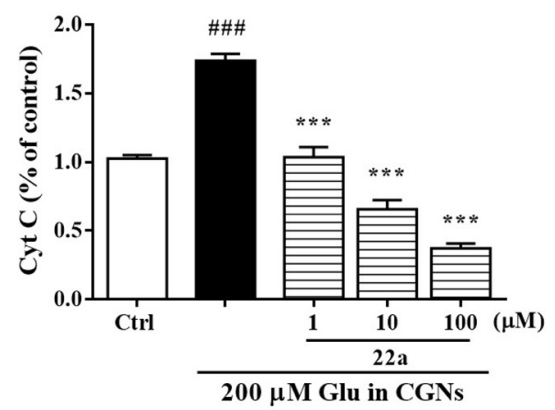

FIGURE 3 | Compound 22a improves mitochondrial dysfunction induced by glutamate. (A) Compound 22 a prevents glutamate-induced changes of MMP in CGNs. CGNs were pre-incubated with various agents for $2 \mathrm{~h}$, then exposed to $200 \mu \mathrm{M}$ glutamate. The MMP was evaluated by staining with the potential sensor JC-1. The fluorescence shift from red to green was detected by a microplate reader. (B) Quantitative analysis of the red fluorescence to green fluorescence (590 $\mathrm{nm} / 529 \mathrm{~nm})$ ratio in (A). (C) Compound 22a attenuates glutamate-induced ATP depletion in CGNs. (D) Immunoblot assay was performed with antibodies against Cyt C.

(E) Densitometric analysis of (D). Data were expressed as the mean \pm SEM of three separate experiments; ${ }^{\# \# \# ~} p=0.001$ versus control group; * $p<0.05$, ** $p<0.01$, and ${ }^{* * *} p<0.001$ versus glutamate group.

measured the MMP collapse and mitochondrial ATP release. Changes of MMP were monitored by a JC-1 molecular probe, and the fluorescence shift from red to green reflected the depolarization of MMP (Chaoui et al., 2006). It was found that pretreatment with compound 22a concentration-dependently and strongly restored MMP decrease (red fluorescence/green fluorescence) in glutamate-treated CGNs (Figures 3A,B). We also found that glutamate induced a significant decrease in mitochondrial ATP production, whereas pretreatment with compound 22a significantly prevented glutamate-induced decreases in ATP levels (Figure 3C). Memantine was also effective ameliorating the decrease of MMP and ATP in glutamate-treated CGNs. In addition, when Cyt C release was measured by Western blot as shown in Figures 3D,E, it was found that 22a concentration-dependently prevented glutamate-induced Cyt $\mathrm{C}$ release.

\section{Compound 22a Up-Regulates PGC1 $\alpha /$ Nrf2 Pathway Through Activation of PI3K/Akt}

PGC1 $\alpha$ was considered to be a major regulator of mitochondrial biogenesis. It is thought to regulate the expression of $\mathrm{Nrf2}$ (Wareski et al., 2009; Robinson et al., 2014), a major transcription 


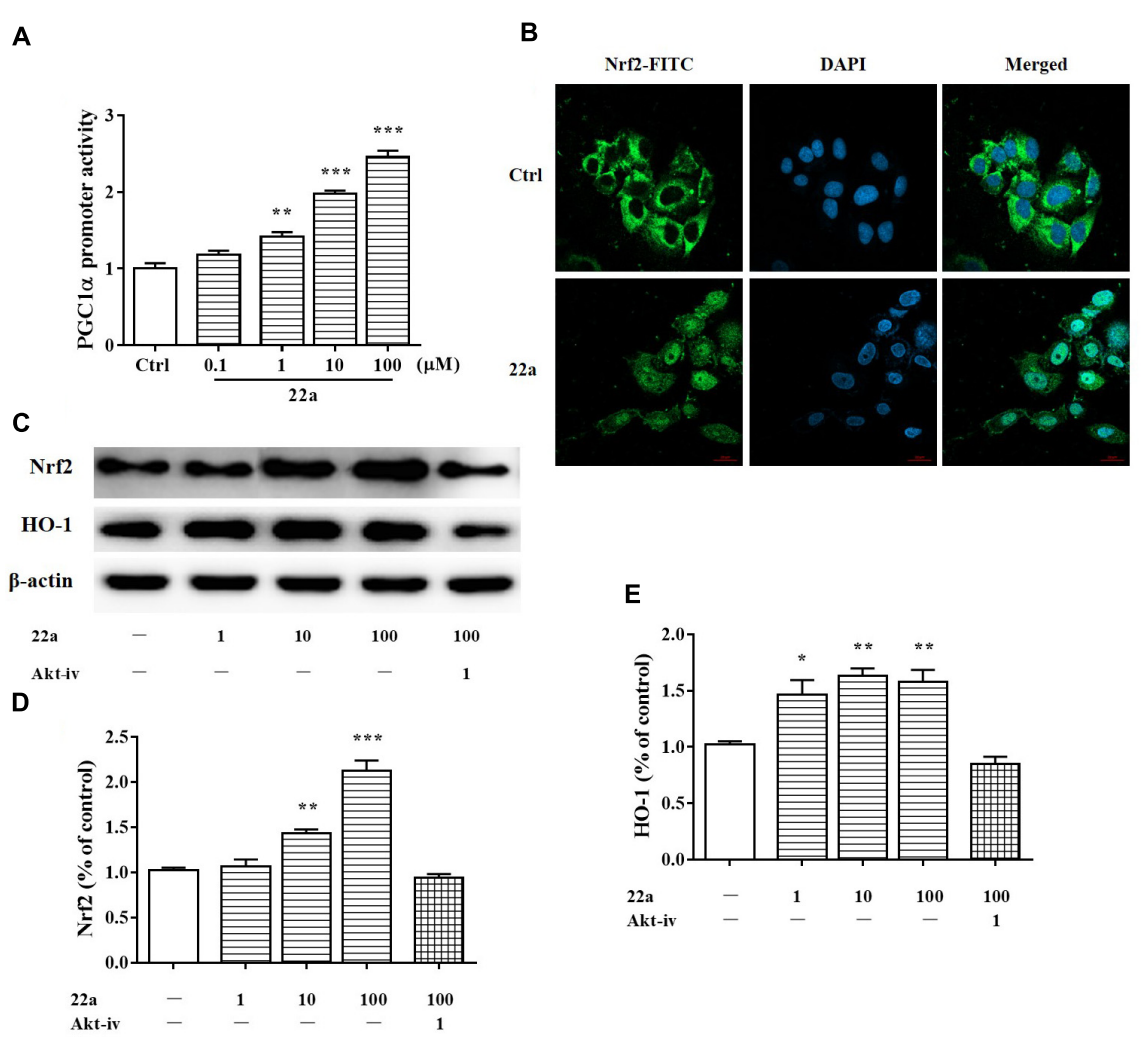

FIGURE 4 | Compound 22a activates of PGC1 $\alpha /$ Nrf2 signaling pathway. (A) Compound 22a $(0.1,1,10,100 \mu \mathrm{M})$ increased the transcriptional activity of PGC1 $\alpha$ determined by the luciferase reporter assay. (B) Compound 22a $(100 \mu \mathrm{M})$ induced the nuclear translocation of Nrf2 determined by immunofluorescence staining. Left panel: green fluorescence showing Nrf2 localization. Middle panel: stained nucleus with DAPI. Right panel: merged images from green and blue filters. Scale bar: $20 \mu \mathrm{m}$. (C) Immunoblot assay was performed with antibodies against Nrf2 and HO-1. (D,E) Densitometric analysis of the protein expression in (C). CGNs were treated with compound 22a $(1,10,100 \mu \mathrm{M})$ for $12 \mathrm{~h}$. Data were expressed as the mean \pm SEM of three separate experiments; ${ }^{*} p<0.05,{ }^{* *} p<0.01$, and ${ }^{* * *} p<0.001$ versus control group.

factor against oxidative stress (Nguyen et al., 2009). As shown in Figure 4A, Compound 22a increased the transcriptional activity of PGC1 $\alpha$ in a concentration-dependent manner. Nrf2 nuclear translocation was further investigated with PC12 cells after treatment with $100 \mu \mathrm{M}$ compound $22 \mathrm{a}$ for $12 \mathrm{~h}$. As shown in Figure 4B, 22a pretreatment increased Nrf2 accumulation in the nucleus. Nrf2 is a crucial regulator against oxidative stress and it is of interest to investigate the involvement of $\mathrm{HO}-1$ protein expression. As shown in Figures 4C-E, CGNs treated with compound 22a concentration-dependently increased Nrf2 and HO-1 expression. PI3K/Akt activation was reported to contribute to the up-regulation of the Nrf2 signal (Lee et al., 2015). We assessed if compound 22a-induced Nrf2 and HO-1 expression was affected by Akt inhibitor Akt-iv. As shown in Figures 4C-E, compound 22a-induced up-regulation of Nrf2 and HO-1 was completely abolished by Akt-iv.

\section{Compound 22a Activates PGC1 $\alpha /$ Nrf2 Signaling Pathway in CGNs Treated With Glutamate}

To further elucidate whether the PGC1 $\alpha / \mathrm{Nrf} 2$ signaling pathways were involved in the protective effect of compound 22a against glutamate-induced neuron injury, the two protein's expression levels were examined with Western blotting. We found that glutamate significantly decreased PGC1 $\alpha, \mathrm{Nrf} 2$, and $\mathrm{HO}-1$ protein expression (Figures 5A,B); however, compound 22a pretreatment reversed these decreases. To confirm the role of PGC1 $\alpha$ activation in the neuroprotective effects of compound 22a, we tested the blocking effect of PGC1 $\alpha$ siRNA transfection. We found that PGC1 $\alpha$ siRNA transfection dramatically decreased PGC1 $\alpha$ protein expression (Figures 5C,D). In cell viability tests, PGC1 $\alpha$ siRNA transfection considerably attenuated the neuroprotection of compound 22a; however, a scrambled RNA transfection that was used as a negative control did not yield any significant effects on PGC1 $\alpha$ expression or on cell viability (Figure 5E).

\section{Compound 22a Reversed the Inhibition of PI3K/Akt/GSK3 $\beta$ Pathway Caused by Glutamate}

To investigate the signaling pathways involved in the protective effects of compound 22a against glutamate-caused excitotoxicity, we evaluated the correlation between cell viability and PI3K/Akt activation. The levels of p-PI3K, p-Ser473-Akt and p-Ser9-GSK3 $\beta$ 

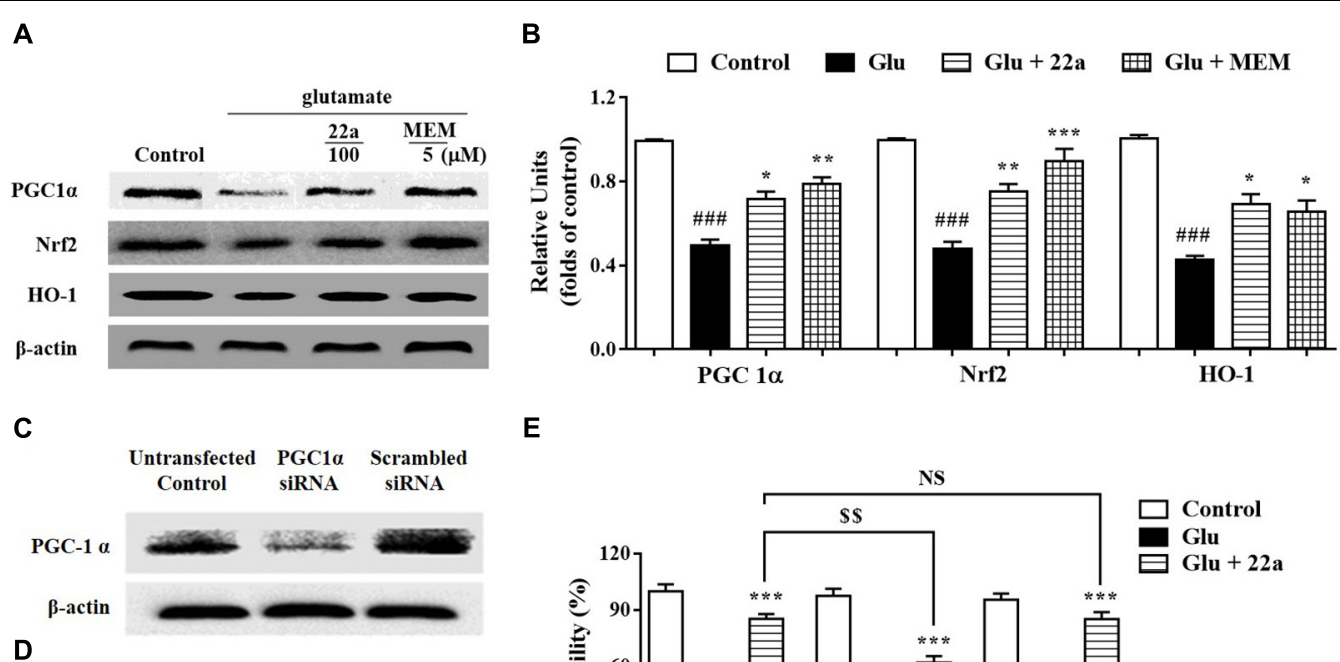

E
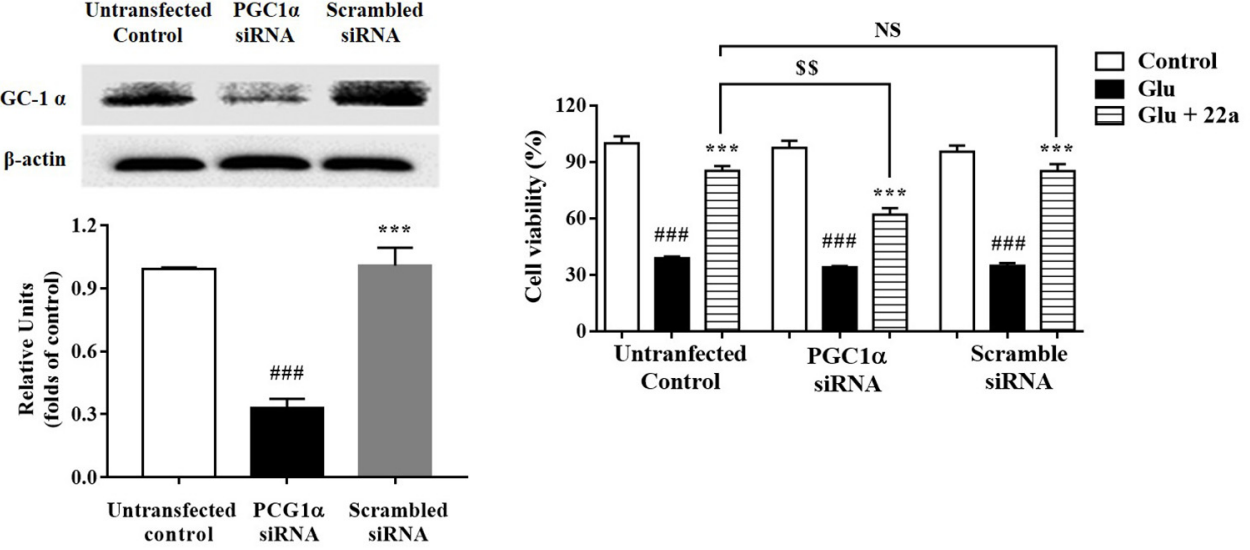

FIGURE 5 | Involvement of the PGC1 1 /Nrf2 pathway in neuroprotection exerted by compound 22a in CGNs. (A) Representative blots showed the expression of proteins PGC1 $\alpha$, Nrf2 and HO-1 in CGNs after different treatments. (B) Densitometric analysis of the protein expression in (A). (C) Representative blots showed the protein expression of PGC1 $\alpha$ was silenced in CGNs pretreated with PGC1 $\alpha$ siRNA, but not when pretreated with scrambled siRNA. (D) Densitometric analysis of (C). (E) PGC1 $\alpha$ siRNA significantly attenuated the neuroprotective effects of compound 22a against glutamate-induced neuronal cell death in CGNs. CGNs were transfected with either PGC1 $\alpha$ siRNA or scrambled siRNA for $24 \mathrm{~h}$ and then pretreated with compound 22a (10 mM) for $2 \mathrm{~h}$ before exposure to $200 \mu \mathrm{M}$ glutamate. Cell viability was measured at $24 \mathrm{~h}$ post glutamate exposure using the MTT assay. Data were expressed as the mean \pm SEM of three experiments; \#\#\# $p<0.01$ versus control group; ${ }^{*} p<0.05,{ }^{* *} p<0.01$, and ${ }^{* * *} p<0.001$ versus glutamate group; NS means no significance; ${ }^{\$} p<0.01$ versus 22 a in the not-transfected control group.

were analyzed by Western blot. As shown in Figures 6AD, glutamate down-regulated the phosphorylation of PI3K and Akt, while pretreatment with both compound 22a and memantine reversed the suppressed phosphorylation of PI3K and Akt. GSK-3 $\beta$ is the molecule downstream of PI3K/Akt and is phosphorylated by the PI3K/Akt pathway. As shown in Figures 6E,F, there was a significant increase in the phosphorylation of GSK-3 $\beta$ after treatment with compound 22a in the presence of glutamate.

\section{PI3K and Akt Inhibitors Attenuate the Neuroprotective Effects of Compound 22a}

Activation of the pro-survival PI3K/Akt signaling pathway has been shown to be important for neuroprotection (Cantrell, 2001). Consistent with our previous study (Xu et al., 2016), when exposing the cells to a PI3K inhibitor, LY294002 $(1 \mu \mathrm{M}), 30 \mathrm{~min}$ prior to the addition of compound 22a, phosphorylation of Akt and GSK3 $\beta$ was nearly completely blocked by LY294002 as shown in Figures 7A-D. Pretreatment with LY294002 reversed compound 22a-prompted Bcl-2 up-regulation and Bax down-regulation; the Bcl-2/Bax ratio was decreased by LY294002 (Figures 7E,F). In line with previous reports that PI3K/Akt is involved in Nrf2 mediated HO-1 expression (Lee et al., 2015), when cells were exposed to an Akt inhibitor, Akt-iv $(1 \mu \mathrm{M})$, 30 min prior to 22a treatment, the up-regulation of PGC1 $\alpha, \mathrm{Nrf} 2$ and HO-1 proteins' expression by compound 22a was almost completely suppressed by Akt-iv, as shown in Figures 7G,H.

To further confirm the involvement of the PI3K/Akt pathway in the neuroprotection exerted by compound 22a in CGNs damaged by glutamate, a specific PI3K inhibitor LY294002 and an Akt inhibitor Akt-iv were applied in a cell viability assay. LY294002 and Akt-iv significantly attenuated the neuroprotection of compound 22a against glutamate toxicity (Figure 7I).

\section{DISCUSSION}

Glutamate is the principal excitatory amino acid neurotransmitter with complex biological activities (PitaAlmenar et al., 2006; Paoletti, 2011). However, a high concentration of extracellular glutamate is toxic to nerve cells 
A

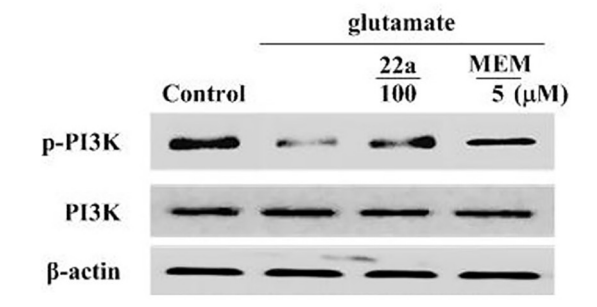

C

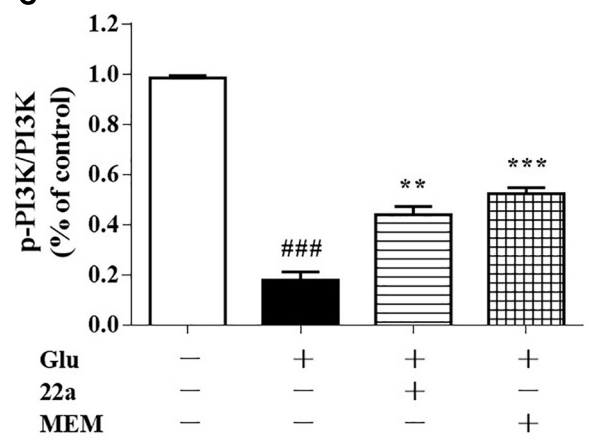

E

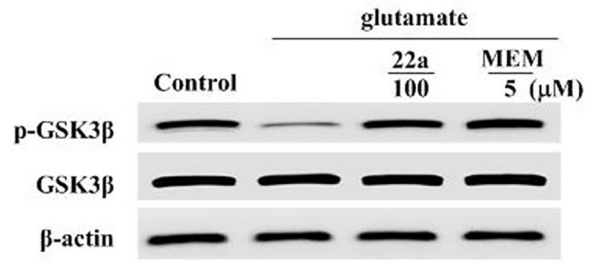

B

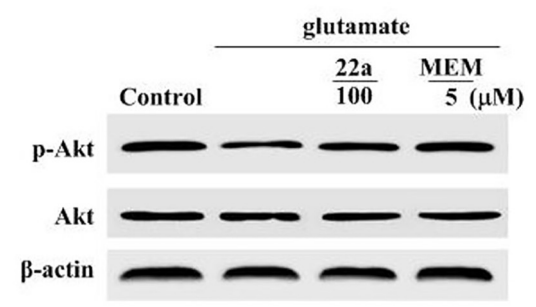

D

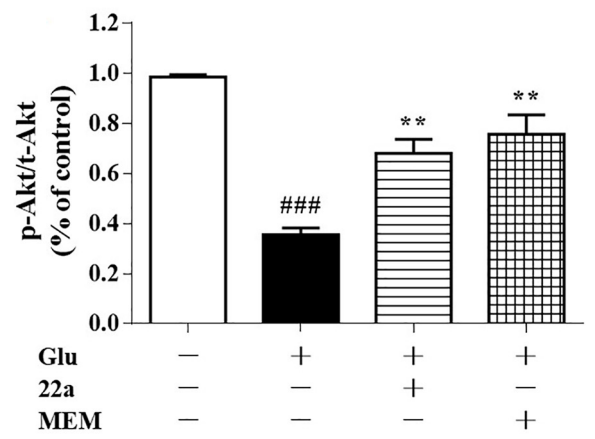

$\mathbf{F}$

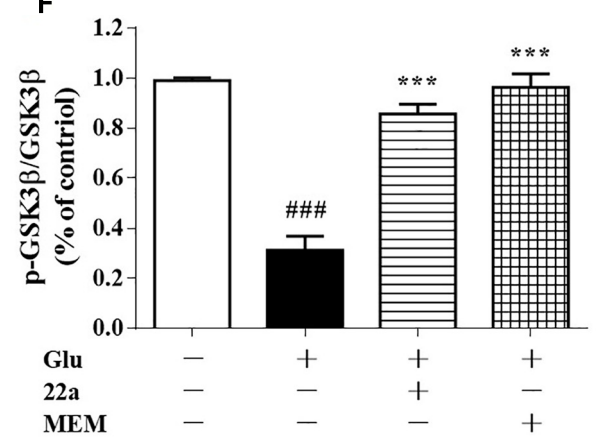

FIGURE 6 | Compound 22a activates PI3K/Akt pathway in glutamate treated CGNs. (A,C,E) Representative blots showed the protein expression of p-PI3K/PI3K (A), p-Akt/Akt (C), and p-GSK3 $\beta / G S K 3 \beta$ (E) in CGNs. CGNs were pretreated with compound 22a and memantine for $2 \mathrm{~h}$ before exposure to glutamate. (B,D,F) Densitometric analysis of the protein expression in (A,C,E). Data were expressed as the mean \pm SEM of three experiments; \#\#\# $p<0.001$ versus control group; ${ }^{* *} p<0.01$ and ${ }^{* * *} p<0.001$ versus glutamate treatment group.

and is considered to be a key contributor in the pathogenesis of neurodegenerative diseases such as ischemic stroke (Wahl et al., 1994). In our previous study, we reported that compound 22a exhibited neuroprotective effects against oxidative stress-induced neuronal loss in vitro and protected against ischemic stroke in vivo (Chen et al., 2017). However, the exact mechanisms underlying the neuroprotection of compound 22a is still unknown. Therefore, the neuroprotective effects of compound 22a against glutamate-induced excitotoxicity were investigated in the current study. We demonstrated that compound 22a protected against glutamate-induced neurotoxicity in CGNs. Meanwhile, we found that compound 22a reversed the MMP collapse and alternation of Bcl-2 and Bax expression to attenuate glutamate-induced cellular apoptosis. Our research further demonstrated that the neuroprotective effects of compound 22a were intermediated by the stimulation of PI3K/Akt and PGC1 $\alpha /$ Nrf2 pathways.
Glutamate is one of the pathological factors in cerebral ischemic disease, and can cause cell apoptosis and MPP reduction, both of which are initiated by the interaction between pro- and anti-apoptotic Bcl-2 family members (Chen Q. et al., 2015). In addition, glutamate toxicity induces mitochondrial dysfunction. Mitochondria are recognized as a center of intracellular energy metabolism, and mitochondrial $\mathrm{Ca}^{2+}$ is a positive effector of ATP synthesis (Feissner et al., 2009). $\mathrm{Ca}^{2+}$ overload, however, results in free radical generation and MPTP opening, which in turn causes mitochondrial depolarization, matrix solute loss, and Cyt $\mathrm{C}$ release (Bernardi and Rasola, 2007). Moreover, the overproduction of ROS is also reported to be related to $\mathrm{mPTP}$ opening (Christophe and Nicolas, 2006). In our study, compound 22a pretreatment significantly prevented intracellular ATP reduction and ROS aggregation, and mitigated MMP dissipation and Cyt C release. Our data further uncovered that compound 22a 


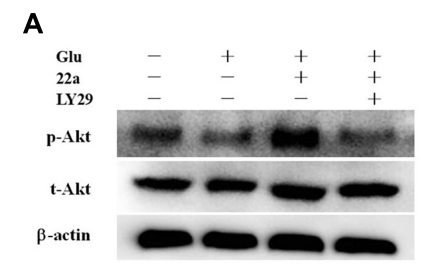

B

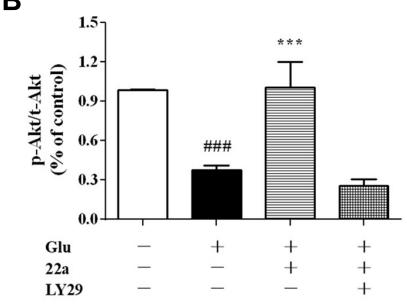

G

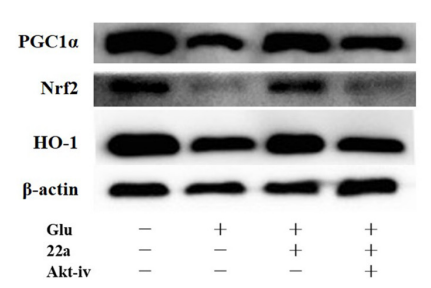

C

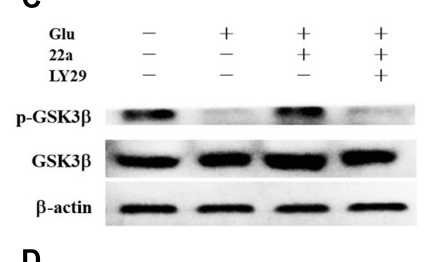

D

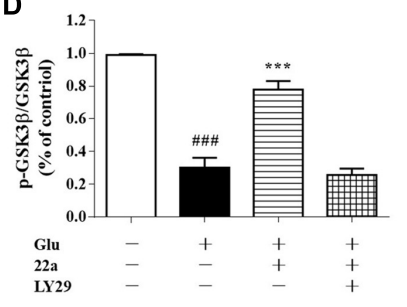

H

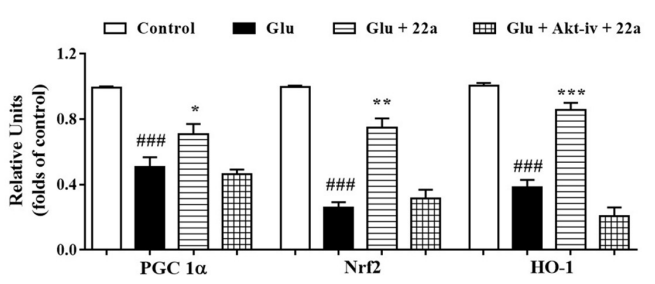

E

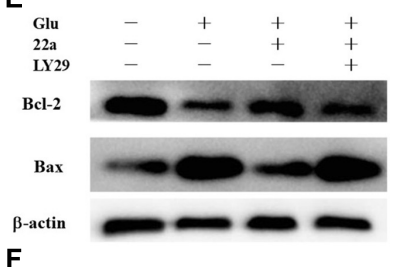

F

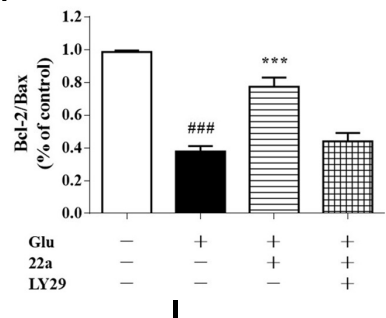

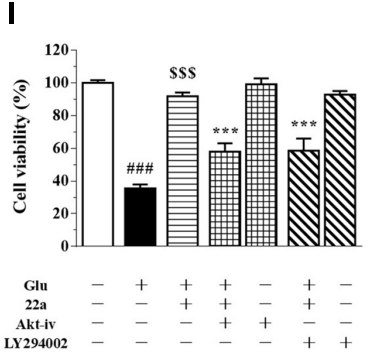

FIGURE 7 | Involvement of the PI3K/Akt pathway in neuroprotection exerted by compound 22a in CGNs. (A,C,E) Representative blots showed the protein expression of p-Akt/Akt (A), p-GSK3 $\beta / G S K 3 \beta$ (C), Bcl-2 and Bax (E). CGNs were pretreated with $1 \mu$ M LY294002 for 30 min before administration of compound 22a (10 mM), followed by glutamate challenge. (B,D,F) Densitometric analysis of the protein expression in (A,C,E). (G) Representative blots showed the protein expression of PGC-1 $\alpha$, Nrf2 and HO-1. (H) Densitometric analysis of the protein expression in (G). (I) Pretreatment with an Akt inhibitor (Akt-iv) and a PI3K inhibitor (LY294002) attenuated the protective effects of compound 22a on glutamate-treated CGNs. Data were expressed as the mean \pm SEM of three experiments; \#\#\# $p<0.001$ versus control group; * $p<0.05$, ${ }^{* *} p<0.01$, and*** $p<0.001$ versus glutamate treatment group; ${ }^{\$ \$ \$} p<0.001$ versus "22a+glutamate" treatment group. LY29 refers to PI3K inhibitor LY294002.

reversed the up-regulation of Bax and down-regulation of Bcl2 expression induced by glutamate. Furthermore, the expression of Bcl-2, an integral membrane protein, is recognized as a hallmark of cell death associated with mitochondria dysfunction (Wallgren et al., 2013). Altogether, these results imply that increased Bcl-2 expression may represent an endogenous repair mechanism against apoptotic pathway, indicating that 22a may block the Bax-mediated decrease of MMP or promote mitochondrial homeostasis against glutamate-caused CGNs damage.

As described above, mitochondria play a vital role in many fundamental cellular processes, ranging from energy production and metabolism to apoptosis (Robinson et al., 2014). PGC1 $\alpha$ is a transcriptional co-activator that regulates the transcription of numerous genes involved in cellular metabolism, including mitochondrial biogenesis and respiration and ROS metabolism (Shin et al., 2011). PGC1 $\alpha$ is also a potent stimulator of mitochondrial respiration and gene transcription that acts by activating nuclear respiratory factors Nrf1 and Nrf2, which in turn regulate expression of mitochondrial transcription factor A (Tfam) and other nuclear-encoded mitochondrial proteins (Wareski et al., 2009). Numerous studies indicate that $\mathrm{Nrf} 2$ combines with the promoter of ARE to create general antioxidant responses, which is recognized as a promising method to therapeutically reestablish the CNS redox balance in neurodegenerative disorders (Lim et al., 2014). Among the enzymes that are redox-sensitive inducible is HO-1, which can protect neurons from acute insults under stress conditions thanks to its antioxidant and anti-inflammatory properties (Chen, 2014). In line with previous studies, we found that compound 22a could enhance PGC1 $\alpha$ transcriptional activity and induce nuclear accumulation of Nrf2 in PC12 cells (Figures 4A,B). In addition, we found that glutamate treatment significantly down-regulated the protein expression of PGC1 $\alpha$ and pretreatment with compound 22a significantly reversed the down-regulated expressions of PGC1 $\alpha, \operatorname{Nrf} 2$ and HO-1 induced by glutamate in CGNs (Figures $\mathbf{5 A}, \mathbf{B}$ ). Several investigations present that $\mathrm{Nrf} 2$ is tightly regulated in neurons through signaling pathways such as PI3K/Akt, which is reported to have a Nrf2-dependent role in activating HO-1 expression (Wang et al., 2008; Zhang et al., 2012; Yin et al., 2015). In this sense, the present study was designed to investigate whether the PI3K/Akt pathway is involved in regulating the $\mathrm{PGC} 1 \alpha$ and $\mathrm{Nrf} 2 / \mathrm{HO}-1$ activation resultant from compound 22a's presence, and what effect that has on compound 22a's subsequent protective against glutamate-induced neurotoxicity. Our results demonstrated that a specific Akt inhibitor significantly suppressed the enhanced 
expression of PGC1 $\alpha, \mathrm{Nrf} 2$ and $\mathrm{HO}-1$ induced by compound 22a (Figures 7G,H). Moreover, to some extent, knockdown of PGC1 $\alpha$ was found to reverse the neuro-protective effect of compound 22a against toxic stress (Figures 5C-E). As such, compound 22a may pave an effective and practical way to modulate PGC1 $\alpha$ activity in neurons.

The PI3K/Akt pathway plays a critical role in preventing the neuronal cell death seen in hypoxic and excitotoxic conditions in vitro. Inhibiting the PI3K/Akt pathway exacerbates ischemic neuronal death (Lai et al., 2014). Previous reports indicate that neuroprotective strategies against glutamateinduced excitotoxicity in the cortex and hippocampus of postnatal brains can trigger the activation of PI3K/Akt (Shah et al., 2014). Additionally, Akt activation is capable of suppressing several pro-apoptotic proteins, including members of the Bcl-2 family and some signaling molecules such as GSK-3 $\beta$ (Maurer et al., 2014). In our study, phosphorylation of PI3K, Akt and GSK3 $\beta$ in CGNs was significantly down-regulated when cells were exposed to glutamate; however, compound 22a pretreatment reversed these changes (Figures 6A-F). In addition, PI3K inhibitor LY29004 significantly inhibited the up-regulation of phosphorylated Akt and GSK3 $\beta$ expression while reversing compound 22ainduced up-regulation of $\mathrm{Bcl}-2$ and down-regulation of Bax expression (Figures 7A-F). Importantly, PI3K and Akt inhibitors completely abolished the neuroprotection conferred by compound 22a pretreatment (Figure 7I). Summarily, our results indicate that $\mathrm{PI} 3 \mathrm{~K} / \mathrm{Akt}$ pathway activation is involved in the neuro-protection of compound 22a against CGNs injury induced by glutamate.

In summary, compound 22a effectively prevented glutamateinduced excitotoxicity of CGNs via involvement of the PI3K/Akt and PGC1 $\alpha / \mathrm{Nrf} 2$ pathways. Our results suggest that compound 22a might be of benefit in preventing neuronal death from ischemic stroke.

\section{REFERENCES}

Bernardi, P., and Rasola, A. (2007). Calcium and cell death: the mitochondrial connection. Subcell. Biochem. 45, 481-506. doi: 10.1007/978-1-4020-6191-2_18 Cantrell, D. (2001). Phosphoinositide 3-kinase signalling pathways. J. Cell Sci. 114, 1439-1445.

Chaoui, D., Faussat, A. M., Majdak, P., Tang, R., Perrot, J. Y., Pasco, S., et al. (2006). JC-1, a sensitive probe for a simultaneous detection of P-glycoprotein activity and apoptosis in leukemic cells. Cytometry B Clin. Cytom. 70, 189-196. doi: $10.1002 /$ cyto.b. 20100

Chen, H., Tan, G., Cao, J., Zhang, G., Yi, P., Yu, P., et al. (2017). Design, synthesis, and biological evaluation of novel tetramethylpyrazine derivatives as potential neuroprotective agents. Chem. Pharm. Bull. 65, 56-65. doi: 10.1248/cpb.c1600699

Chen, H. Y., Xu, D. P., Tan, G. L., Cai, W., Zhang, G. X., Cui, W., et al. (2015). A potent multi-functional neuroprotective derivative of tetramethylpyrazine. J. Mol. Neurosci. 56, 977-987. doi: 10.1007/s12031-015-0566-x

Chen, Q., Xu, H., Xu, A., Ross, T., Bowler, E., Hu, Y., et al. (2015). Inhibition of $\mathrm{Bcl}-2$ sensitizes mitochondrial permeability transition pore (MPTP) opening in ischemia-damaged mitochondria. PLoS One 10:e0118834. doi: 10.1371/journal. pone. 0118834

Chen, J. (2014). Heme oxygenase in neuroprotection: from mechanisms to therapeutic implications. Rev. Neurosci. 25, 269-280. doi: 10.1515/revneuro2013-0046

\section{ETHICS STATEMENT}

All animal studies were conducted following to the handbook of the Experimental Animal Care and Use Committee of Jinan University, and the experimental protocols were approved by the Ethics Committee for Animal Experiments of Jinan University.

\section{AUTHOR CONTRIBUTIONS}

HC conceptualized and designed the experiments, performed the research, analyzed and interpreted the results, and wrote the manuscript. JC and ZyZ helped design and performed the experiments. YW, ZjZ, YS, GZ, LS, and PY conceptualized and designed the experiments, analyzed and interpreted the results, and revised the manuscript.

\section{FUNDING}

This work was partially supported by the Natural Science Fund of China (81502908), the China Postdoctoral Science Foundation (2017M622646), the Science and Technology Project of Guangdong Province (2013A022100030, 2014A030310174, 2015A020211019, 2015B020232011, 2016A020217013, and 2016A030313107), GD-HK Cooperative Project (2016A050503030), the Key Laboratory Project of Guangzhou (151800010), the Science and Technology Program of Guangzhou (201704020181); as well as the Fundamental Research Funds for the Central Universities (21617469).

\section{ACKNOWLEDGMENTS}

We thank Ms. Linda Wang for proof-reading the manuscript.

Christophe, M., and Nicolas, S. (2006). Mitochondria: a target for neuroprotective interventions in cerebral ischemia-reperfusion. Curr. Pharm. Des. 12, 739-757. doi: 10.2174/138161206775474242

Donnan, G. A., Fisher, M., Macleod, M., and Davis, S. M. (2008). Stroke. Lancet 371, 1612-1623. doi: 10.1016/S0140-6736(08)60694-7

Du, Y., Bales, K. R., Dodel, R. C., Hamilton-Byrd, E., Horn, J. W., Czilli, D. L., et al. (1997). Activation of a caspase 3-related cysteine protease is required for glutamate-mediated apoptosis of cultured cerebellar granule neurons. Proc. Natl. Acad. Sci. U.S.A. 94, 11657-11662. doi: 10.1073/pnas.94.21.11657

Feissner, R. F., Skalska, J., and Gaum, W. E. (2009). Crosstalk signaling between mitochondrial Ca2+ and ROS. Front. Biosci. 14:1197-1218. doi: 10.2741/3303

Floyd, R. A., Castro, Faria Neto HC, Zimmerman, G. A., Hensley, K., and Towner, R. A. (2013). Nitrone-based therapeutics for neurodegenerative diseases: their use alone or in combination with lanthionines. Free Radic. Biol. Med. 62, 145-156. doi: 10.1016/j.freeradbiomed.2013.01.033

Ghosh, N., Ghosh, R., Bhat, Z. A., Mandal, V., Bachar, S. C., Nima, N. D., et al. (2014). Advances in herbal medicine for treatment of ischemic brain injury. Nat. prod. commun. 9, 1045-1055.

Grupke, S., Hall, J., Dobbs, M., Bix, G. J., and Fraser, J. F. (2014). Understanding history, and not repeating it. Neuroprotection for acute ischemic stroke: from review to preview. Clin. Neurol. Neurosurg. 129, 1-9. doi: 10.1016/j.clineuro. 2014.11.013

Jing, Y., Liu, L. Z., Jiang, Y., Zhu, Y., Guo, N. L., Barnett, J., et al. (2012). Cadmium increases HIF-1 and VEGF expression through ROS, ERK, and AKT signaling 
pathways and induces malignant transformation of human bronchial epithelial cells. Toxicol. Sci. 125, 10-19. doi: 10.1093/toxsci/kfr256

Jo, H., Mondal, S., Tan, D., Nagata, E., Takizawa, S., Sharma, A. K., et al. (2012). Small molecule-induced cytosolic activation of protein kinase Akt rescues ischemia-elicited neuronal death. Proc. Natl. Acad. Sci. U.S.A. 109, 10581-10586. doi: 10.1073/pnas.1202810109

Kim, H. K., Song, I. S., Lee, S. Y., Jeong, S. H., Lee, S. R., Heo, H. J., et al. (2014). B7-H4 downregulation induces mitochondrial dysfunction and enhances doxorubicin sensitivity via the CAMP/CREB/PGC1- $\alpha$ signaling pathway in HeLa cells. Pflugers Arch. 466, 2323-2338. doi: 10.1007/s00424-014-1493-3

Lai, T. W., Zhang, S., and Wang, Y. T. (2014). Excitotoxicity and stroke: identifying novel targets for neuroprotection. Prog. Neurobiol. 115, 157-188. doi: 10.1016/ j.pneurobio.2013.11.006

Lau, A., and Tymianski, M. (2010). Glutamate receptors, neurotoxicity and neurodegeneration. Pflugers Arch. 460, 525-542. doi: 10.1007/s00424-0100809-1

Lee, D. S., Cha, B. Y., Woo, J. T., Kim, Y. C., and Jang, J. H. (2015). Acerogenin a from acer nikoense maxim prevents oxidative stress-induced neuronal cell death through Nrf2-mediated heme oxygenase-1 expression in mouse hippocampal HT22 cell line. Molecules 20, 12545-12557. doi: 10.3390/ molecules 200712545

Lim, J. L., Wilhelmus, M. M., de, Vries HE, Drukarch, B., Hoozemans, J. J., and van, Horssen J (2014). Antioxidative defense mechanisms controlled by Nrf2: state-of-the-art and clinical perspectives in neurodegenerative diseases. Arch. Toxicol. 88, 1773-1786. doi: 10.1007/s00204-014-1338-z

Liu, X., Zhang, R., Xu, W., Li, C., Zhao, Q., and Wang, X. (2003). Synthesis of the novel liqustrazine derivatives and their protective effect on injured vascular endothelial cell damaged by hydrogen peroxide. Bioorg. Med. Chem. Lett. 13, 2123-2126. doi: 10.1016/S0960-894X(03)00359-7

Maurer, U., Preiss, F., Brauns-Schubert, P., Schlicher, L., and Charvet, C. (2014). GSK-3 - at the crossroads of cell death and survival. J. Cell Sci. 127, 1369-1378. doi: $10.1242 /$ jcs. 138057

Nguyen, T., Nioi, P., and Pickett, C. B. (2009). The Nrf2-antioxidant response element signaling pathway and its activation by oxidative stress. J. Biol. Chem. 284, 13291-13295. doi: 10.1074/jbc.R900010200

Pang, T., Wang, Y. J., Gao, Y. X., Xu, Y., Li, Q., Zhou, Y. B., et al. (2016). A novel GSK-3 $\beta$ inhibitor YQ138 prevents neuronal injury induced by glutamate and brain ischemia through activation of the Nrf2 signaling pathway. Acta Pharmacol. Sin. 37, 741-752. doi: 10.1038/aps.2016.3

Paoletti, P. (2011). Molecular basis of NMDA receptor functional diversity. Eur. J. Neurosci. 33, 1351-1365. doi: 10.1111/j.1460-9568.2011.07628.x

Pita-Almenar, J. D., Collado, M. S., Colbert, C. M., and Eskin, A. (2006). Different mechanisms exist for the plasticity of glutamate reuptake during early long-term potentiation (LTP) and late LTP. J. Neurosci. 26, 10461-10471. doi: 10.1523/JNEUROSCI.2579-06.2006

Robinson, A., Grösgen, S., and Mett, J. (2014). Upregulation of PGC-1 $\alpha$ expression by Alzheimer's disease-associated pathway: presenilin 1/amyloid precursor protein (APP)/intracellular domain of APP. Aging Cell 13, 263-272. doi: $10.1111 /$ acel.12183

Sano, M., and Fukuda, K. (2008). Activation of mitochondrial biogenesis by hormesis. Circ. Res. 103, 1191-1193. doi: 10.1161/CIRCRESAHA.108.189092

Shah, S. A., Lee, H. Y., Bressan, R. A., Yun, D. J., and Kim, M. O. (2014). Novel osmotin attenuates glutamate-induced synaptic dysfunction and neurodegeneration via the JNK/PI3K/Akt pathway in postnatal rat brain. Cell Death Dis. 5, e1026. doi: 10.1038/cddis.2013.538

Shin, J. H., Ko, H. S., Kang, H., Lee, Y., Lee, Y. I., Pletinkova, O., et al. (2011). PARIS (ZNF746) repression of PGC-1 $\alpha$ contributes to neurodegeneration in
Parkinson's disease. Cell 144, 689-702. doi: 10.1016/j.cell.2011.02.010S00928674(11)00124-3

Touaibia, M., Jean-Francois, J., and Doiron, J. (2011). Caffeic Acid, a versatile pharmacophore: an overview. Mini Rev. Med. Chem. 11, 695-713. doi: 10.2174/ 138955711796268750

Wahl, F., Obrenovitch, T. P., Hardy, A. M., Plotkine, M., Boulu, R., and Symon, L. (1994). Extracellular glutamate during focal cerebral ischaemia in rats: time course and calcium dependency. J. Neurochem. 63, 1003-1011. doi: 10.1046/j. 1471-4159.1994.63031003.x

Wallgren, M., Lidman, M., Pedersen, A., Brännström, K., Karlsson, B. G., and Gröbner, G. (2013). Reconstitution of the anti-apoptotic Bcl-2 protein into lipid membranes and biophysical evidence for its detergent-driven association with the pro-apoptotic bax protein. PLoS One 8:e61452. doi: 10.1371/journal.pone. 0061452

Wang, L., Chen, Y., Sternberg, P., and Cai, J. (2008). Essential roles of the PI3 kinase/Akt pathway in regulating Nrf2-dependent antioxidant functions in the RPE. Invest. Ophthalmol. Vis. Sci. 49, 1671-1678. doi: 10.1167/iovs.071099

Wang, Y., and Qin, Z. H. (2010). Molecular and cellular mechanisms of excitotoxic neuronal death. Apoptosis 15, 1382-1402. doi: 10.1007/s10495-010-0481-0

Wareski, P., Vaarmann, A., Choubey, V., Safiulina, D., Liiv, J., Kuum, M., et al. (2009). PGC- $1 \alpha$ and PGC-1 $\beta$ regulate mitochondrial density in neurons. J. Biol. Chem. 284, 21379-21385. doi: 10.1074/jbc.M109.018911

Xu, D., Chen, H., Mak, S., Hu, S., Tsim, K. W. K., Hu, Y., et al. (2016). Neuroprotection against glutamate-induced excitotoxicity and induction of neurite outgrowth by T-006, a novel multifunctional derivative of tetramethylpyrazine in neuronal cell models. Neurochem. Int. 99, 194-205. doi: 10.1016/j.neuint.2016.07.006

Xue, Y., Tie, C. R., Li, J., Tian, T., and Li, Q. X. (2011). Ligustrazine inhibits lipopolysaccharide-induced proliferation by affecting P27, Bcl-2 expression in rat mesangial cells. Eur. J. Pharmacol. 665, 8-12. doi: 10.1016/j.ejphar.2011. 05.004

Yin, X., Wang, X., Fan, Z., Peng, C., Ren, Z., Huang, L., et al. (2015). Hyperbaric oxygen preconditioning attenuates myocardium ischemia-reperfusion injury through upregulation of heme oxygenase 1 expression: PI3K/Akt/Nrf2 pathway involved. J. Cardiovasc. Pharmacol. Ther. 20, 428-438. doi: 10.1177/ 1074248414568196

Zhang, J., Li, Y., Chen, X., Pan, Y., Zhang, S., and Wang, Y. (2014). Systems pharmacology dissection of multi-scale mechanisms of action for herbal medicines in stroke treatment and prevention. PLoS One 9:e102506. doi: 10.1371/journal.pone.0102506

Zhang, R., Chae, S., Lee, J. H., and Hyun, J. W. (2012). The cytoprotective effect of butin against oxidative stress is mediated by the up-regulation of manganese superoxide dismutase expression through a PI3K/Akt/Nrf2dependent pathway. J. Cell. Biochem. 113, 1987-1997. doi: 10.1002/jcb.24068

Conflict of Interest Statement: The authors declare that the research was conducted in the absence of any commercial or financial relationships that could be construed as a potential conflict of interest.

Copyright $\odot 2018$ Chen, Cao, Zhu, Zhang, Shan, Yu, Wang, Sun and Zhang. This is an open-access article distributed under the terms of the Creative Commons Attribution License (CC BY). The use, distribution or reproduction in other forums is permitted, provided the original author(s) and the copyright owner $(s)$ are credited and that the original publication in this journal is cited, in accordance with accepted academic practice. No use, distribution or reproduction is permitted which does not comply with these terms. 